\title{
Unusual Site of Metastasis of Bronchogenic Carcinoma
}

Pant $\mathrm{P}^{1}$, Sedhain $\mathrm{A}^{2}$, D'Souza $\mathrm{S}^{3}$, Renuka BG ${ }^{4}$

${ }^{1}$ Department of Internal Medicine

Dhulikhel Hospital - Kathmandu University Hospital,

Kavre, Nepal

${ }^{2}$ Department of Internal Medicine

College of Medical Science,

Bharatpur, Nepal

${ }^{3,4}$ Department of Internal Medicine

Kasturba Medical College,

Mangalore, India

\section{Correspondinvg Author}

Dr Pankaj Pant

Department of Internal Medicine

Dhulikhel Hospital - Kathmandu University Hospital,

Kavre, Nepal

Email.drpant77@yahoo.com

\begin{abstract}
Metastasis of bronchogenic carcinoma to the chest wall and axillary lymphnodes is a rare occurence. This study reports the case of a patient presenting with chest wall swelling as initial symptom which on evaluation was found to be a lymphnode metastasis. The patient also had axillary lymphnode metastasis on the same side as the chest swelling with a contralateral pleural effusion. Here, we discuss the pathways and possible mechanisms of contra lateral auxillary and chest wall lymphnode involvement without ispilateral nodal involvement in bronchogenic carcinoma.
\end{abstract}

\section{Key Words}

bronchogenic carcinoma, contralateral metastasis, axillary lymphnodes, chest wall mass

Kathmandu Univ Med J 2010;9(32):420-2

\section{INTRODUCTION}

Bronchogenic carcinoma may be clinically dormant in many cases until the involvement of pleura or erosion ofbonesoccurs. However,focalchestwallswelling with axillarylympnodeasaninitial presentiog of symptoms of bronchogenic carcinoma is quite rare. It often poses diagnosticchallengesasithastobedifferentiatedfrom numerous otherbutequallyless common conditions. ${ }^{1}$ Contralateral axillaryelymphnode and chest wall involovementwithoutipsilateralnodalinvolvementin bronchogeniccarcinimaasdocumentedinourcaseisquite rareandthepossiblehypotheseisforsuchapresentation and their implications on treatment and prognosis are discussed.

\section{CASE REPORT}

A 58 year old man, presented ato our hospital with complaintsofprogressivebreathlessnessandswellingin theleftchestandaxilla.Hefirstnoticedapainlessswelling threemonthsbackwhich hadgraduallyincreasedinsize. The patient also gave ae history of cough with streaky hemoptysis.
On examination, an oval swelling of $7 \times 5 \mathrm{~cm}$ was noted in the left anterior chest wall at the infraclavicular area overlying the second to fourth ${ }^{\mathrm{h}}$ ribs. A second swelling sized $\mathrm{cm}$ was also seen in the left axilla (Figure-1). The swellingswerenontender,firminconsistency, irreducibl, with restricted mobility, no impulse on coughing, and the skin over the swelling was normal. Respiratory examination revealed that there was a stony dull note with absent breathing sounds on the right side of the chest.AFfrontalchestradiographshowedamassiveright sided pleuraleffusion.AContrastEnhancedComputed Tomography(CECT) ofthechestshowedthattherewasa rightmainbronchusendoluminalmasscausingbronchial cutoffandcollapse(Figure-2). Therewasalso evidence ofextensivemediastinallymphadenopathywithmassive right sided pleural effusion and multiple sub pleural nodules suggestive of pleural metastases.

FNAC of the left infraclavicular mass and axillary mass showed metastatic well differentiated squamous cell carcinoma.Thepatientwasnottakenupforbronchoscopy and biopsydue to his poor performance status. Hewas treatedsymptomaticallywiththoracocentesis.ThePpatient andhisrelativesdeniedfurthertreatmentinviewofpoor prognosis. 


\section{DISCUSSION}

Cancersmainlyspreadbythreemechanisms,localinvasion; lymphaticstream;and/orhaematogenousspreading.The mechanismofspreaddependsontheparticulartumour subtypeandlocation.Althoughtumourscancreatetheir own blood supplybythe process ofangiogenesis, they do not have their own lymphatic drainage and spread is initially by local invasion of surrounding lymphatics. Malignantcellsmayalsobescavengeddirectlyfromthe interstitialtissuesbysurroundinglymphatics. Theymay thendrainintoregionalelymphnodespriortoanydirect vascularinvasionbythetumours. Mostofthesecellswill die but some may have the ability to survive and grow inaenewenvironment.Thisdependsontheunderlying cellpropertiesandgeneticsoftheparticulartumourtype. Shouldthecellsurvivs, atumourgrowsintheelymphnode and may then progress to subsequent nodes.

The tumour may also locally invade beyond theelymphnodecapsule.Onceaelymphnodeiscompletely invadedbythetumour,theusualelymphnodedrainageof theregionwillbedisrupted, whichmayresultinretrograde lymphaticspread. ${ }^{2}$ This was perhaps themechanism in ourcasewherein thecontralateralchestwallandnodal metaatasisprobablyresulted fromatumourblockageof lymphaticsandretrogradespread.Axillaasanunusualsite ofmetastasishasbeenreported withanincidenceof $6.6 \%$ inbronchogeniccarcinoma. ${ }^{3}$ Thecommonestprimarysite with contr lateral spread is the right upper lobe. ${ }^{4}$
Bronchogeniccarcinomainvolvingachestwallinvasion canbeexplained bythefact thatatumouralspreadmay occurthroughnewlydevelopedlymphaticchannelsasa result of pleural adhesions.

Axillaryelymphnodemetastasesmaybeinvolvedthrough adirectchestwallinvasion ofbronchogeniccarcinoma orretrogradespreadfromasupraclavicularlymphnode block. ${ }^{5}$ Withsupportivecaremeasures, themediansurvival rateof patientsattheadvancedstageofthediseaseis 16 to 17 weeks.Althoughchemotherapyisthebackboneof treatmentformetastaticdiseases,theresponseratesarelow, andsurvivaltimesarepoor.Howeverstudiesshowplatinu basedregimensimprovesymptoms, and cancontroland increasntheone-yearsurvival ratechancebetween 10\% and $20 \%$.t.Thebenefitsoftherapyareusuallyrestrictedto otherwisehealthypatientswithlungcancer,suchasthose who maintain a good functional status. ${ }^{6}$

Sincetheexpectedoverallsurvivaltimeislow,minimizing hospitalizationandcausingminimaldistressareimportant factors when determining the treatment. ${ }^{7}$

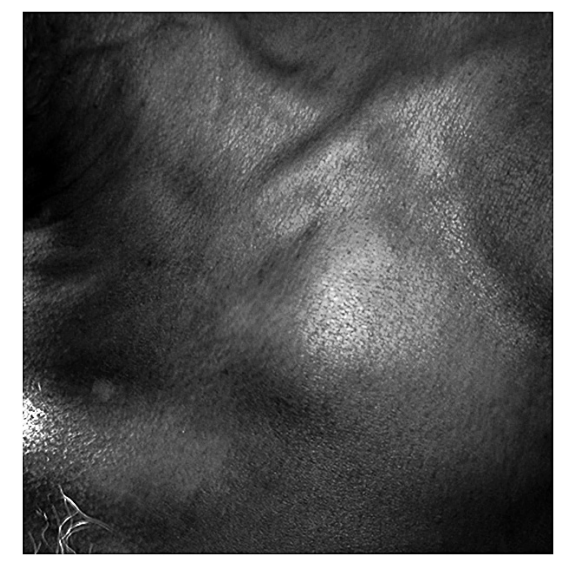

Figure 1. Left chest wall swelling

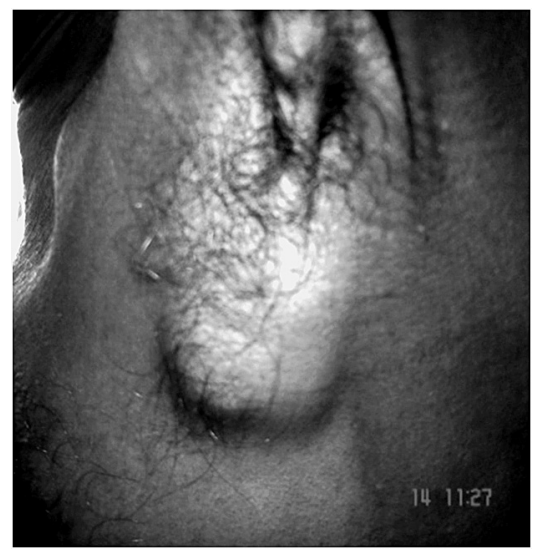

Figure 2. Left axillary mass 


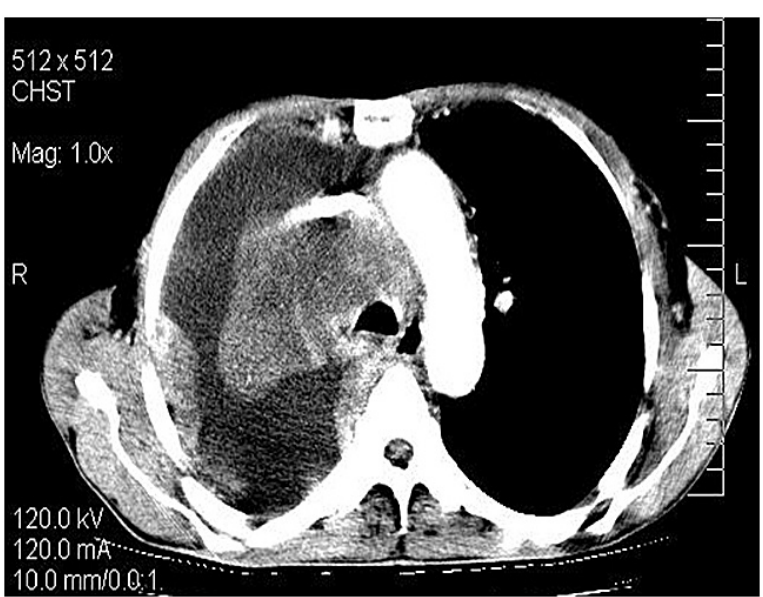

Figure 3. CECT showing right main bronchus endoluminalmasswithcollapseandmassiveright seded pleural effusion

\section{REFERENCES}

VishakAK,KamalakshSK,JayaramaSKetal.Chestwallmassof rare aetiology. J Ind Acad Clin Med. 2005; 6 (2):164- 6.

GrahamR,Cherryman,Bruno,Morgan.LymphaticSystem.In :DavidSutton-TextbookofRadiologyandlmaging.Vol1.7thed. Churchill Livingstone ,2002:511- 512.

MarcantonioDR,LibshitzHI.Axillarylymphnodemetastases of bronchogenic carcinoma. Cancer 1995;76:803- 6.

Baird JA. The pathways of lymphatic spread of carcinoma of lung.Br j surg1965;17:201-4.

Marc R, Françoise Le PB, Claire D. Axillary lymphnode metastases from bronchogenic carcinoma. Ann Thorac surg1998;66:920-2.

AlexanderS,DavidSE.MultidisciplinaryManagementofLung Cancer.N Engl J Med 2004; 350:379-92.

FrankA, Baciewicz. Malignant pleural effusion. In: HarveyIP, JamesBM,DavidHJetal-LungCancerprinciplesandpractice ${ }^{\text {nd }}$ ed. Lippincott Williams and Wilkins, 2000:1030-31. 